\title{
Retorno de la Educación Superior en Chile. Efecto en la movilidad social a través del estimador de Diferencias en Diferencias
}

\author{
Luis R. Améstica ${ }^{(1)}$, Xavier Llinas-Audet ${ }^{(2)}$ y Iván R. Sánchez ${ }^{(3)}$ \\ (1) Departamento Gestión Empresarial, Facultad de Ciencias Empresariales, Universidad del Bío-Bío, \\ Casilla 5C, Concepción-Chile. (e-mail: lamestica@ubiobio.cl) \\ (2) Catedra UNESCO de Dirección Universitaria, Universidad Politécnica de Cataluña, \\ Campus Nord - Edif. C5, C. Jordi Girona, 1- 3, 08034 Barcelona-España. (e-mail: xavier.llinas@upc.edu) \\ (3) Departamento de Física, Facultad de Ciencias, Universidad del Bío-Bío, Casilla 5C, Concepción-Chile \\ (e-mail: isanchez@ubiobio.cl)
}

Recibido Nov. 22, 2013; Aceptado Ene. 28, 2014; Versión final recibida Mar. 25, 2014

\begin{abstract}
Resumen
El presente trabajo analiza la evolución de la tasa de retorno a la educación terciara en Chile, utilizando la técnica del estimador de "Diferencias en Diferencias". El estudio busca establecer el impacto que ésta genera en la movilidad social, utilizando datos de corte transversal obtenidos de la encuesta de caracterización socioeconómica gubernamental (Casen), para los períodos 2003 a 2009 y 2006 a 2011. Este estudio contribuye con nueva evidencia al confirmar que el salario de las personas más su nivel de estudios puede dar respuestas a hipótesis que podrían explicar un cambio en la estratificación social de los individuos. Para el período 2003 a 2009, la tasa interna de retorno de la educación superior es positiva. Sin embargo, se evidenció que no hay mejoramiento real de ingresos para el individuo que decidió estudiar en el período 2006 a 2011.
\end{abstract}

Palabras clave: retorno, salarios, diferencias en diferencias, movilidad social, educación superior

\section{Return of Higher Education in Chile. Effect on Social Mobility through Differences-in-Differences estimator}

\begin{abstract}
This paper analyzes the evolution of return rates of tertiary education in Chile using differences-indifferences estimation. The study seeks to establish the impact of these rates on social mobility using crosssectional data obtained from the national socioeconomic characterization survey (Casen) for the periods 2003 to 2009 and 2006 to 2011 . This study provides new evidence by confirming that salaries plus the level of education of people could explain a change in the social stratification of individuals. For the period 2003 to 2009, the internal return rate of higher education is positive. However, no real improvement in income is observed of a person who decided to study in the period 2006-2011.
\end{abstract}

Keywords: return, salaries, differences in differences, social mobility, higher education 


\section{INTRODUCCION}

La Educación Superior se ha transformado en un factor estratégico de las políticas sociales y de desarrollo de los países, convirtiéndose en un motor de la movilidad social (Urzúa, 2012) y en la herramienta más efectiva para superar la pobreza y enfrentar la desigualdad, es la educación (Behrman, 2011), la cual afecta la productividad laboral y, por ende, explica los niveles salariales de la población. Para la mayoría de los países, se aprecia una correlación positiva entre inversión en capital humano y salarios laborales (Carnoy, 2011). Asimismo, las diferencias a nivel individual en los recursos de capital humano, siguen siendo fuente primaria de las desigualdades sociales, en las sociedades modernas (Tieben, Hofäcker, y Biedinger, 2013).

La literatura económica, ha utilizado una herramienta que ha denominado "retornos a la educación" que, en términos generales, mide la variación en los ingresos como resultado de la adquisición de un cierto nivel educativo o del incremento de un año de escolaridad. La manera típica de analizar esta relación es mediante las ecuaciones "mincerianas", que se basa en el cálculo de las tasas de retorno (Sapelli, 2011). Se formula un modelo matemático, que relaciona las ganancias en el mercado de trabajo con las inversiones en capital humano. La ecuación de ingresos por capital humano (Mincer, 1974), relaciona los ingresos laborales con las inversiones realizadas en capital humano, aproximadas con los años de educación y años de experiencia laboral.

Ahora bien, es una preocupación de la política pública impulsada por los gobiernos, medir el premio en ingreso asociado con la adquisición de mayor capital humano, considerándolo un elemento clave para el progreso económico y social. Se ha planteado la existencia de varios factores que influyen en su desarrollo, como son la familia, las escuelas, las empresas, origen étnico, entre otros; aunque en general, la discusión sobre el capital humano se centra en la escolaridad como el principal productor de habilidades (Carneiro \& Heckman, 2003). La inversión en capital humano adicional, haría más productivo a un trabajador en el futuro y, por tanto, le permitiría tener un mayor potencial de ingresos.

Desde una perspectiva privada, los retornos a la educación proporcionan una medida por invertir en escolaridad adicional. Asimismo, desde un punto de vista social, puede ser un indicador de la relativa escasez de personas con diferentes niveles educativos y, en consecuencia, ser una guía de políticas públicas. La teoría del capital humano, postula que la educación es una actividad de inversión que aumenta la productividad del trabajo y los ingresos de por vida, de los individuos (Hung, 2008). La rentabilidad económica de la educación se puede medir por la tasa interna de retorno a la educación, que es la tasa de interés que iguala el valor presente de los costos incurridos para la educación, al valor presente de los ingresos de por vida adicionales atribuibles a la educación. La tasa de retorno de la educación, captura el efecto sobre los ingresos que tiene un año adicional de educación, resultando evidente que ésta no es la misma para cada nivel de educación: primaria, secundaria o superior.

No obstante la educación, también puede ser vista como una inversión que genera externalidades (Canton, 2007; y Moretti, 2004), porque no sólo aumenta la productividad del factor trabajo sino la de todos los demás factores disponibles.(Psacharopoulos y Patrinos 2004). También se le ve como un bien público, que contribuye a la sociedad, mejorando el capital humano y el desarrollo económico, aunque últimamente ha comenzado a ser considerado como un bien privado (Altbach, Reisberg, y Rumbley, 2009; Pedraja, Araneda, Rodríguez, y Rodríguez, 2012). Finalmente, la educación puede verse como un bien señalizador, según lo propuesto por Hungerford y Solon (1987), donde la titulación marca la existencia de habilidades en los estudiantes, señalizando el hecho de que tienen esas habilidades (en Münich et al. 2005). Se entiende que, en Chile, existe la posibilidad que una persona se movilice socialmente a través del logro individual, siendo la educación superior factor predominante para ascender en la estructura social (Torche y Wormald, 2004). Por ello, es fundamental el rol del Estado en el desarrollo de la educación, en pos de generar movilidad social y ayudar en la formación de los ciudadanos para la sociedad (Pedraja et al., 2012).

En el trabajo propuesto se usará el estimador de diferencia en diferencias, método alternativo y convergente a Mincer, a través de la estimación de la inferencia causal estadística, en el contexto de estudios observacionales (Heckman, Ichimura, Smith, y Todd, 1998), comparando la diferencia en el comportamiento promedio antes y después de la reforma, para el grupo de elegibles contrastándolo con el grupo de comparación (Carneiro, Heckman, y Vytlacil, 2010). Este trabajo desarrolla un ejercicio de evaluación, basado en la utilización del estimador de diferencias en diferencias, para lo cual se utiliza la metodología empleada por (Abadie, 2005). El objetivo de la investigación es entregar una aproximación a movilidad social, utilizando el software Stata con bases de datos de la encuesta de caracterización socioeconómica en Chile (CASEN), principal instrumento de medición del diseño de evaluación de las políticas sociales que existen en dicho país (MIDEPLAN, 2011). 
Por lo tanto, el modelo propuesto, permite extrapolar las conclusiones al total de la población - objetivo, es decir, individuos que han obtenido un título en educación superior (universidad, instituto profesional o centro de formación técnica), comparando dos bases, los períodos: 2003-2009 y 2006-2011, cuyos datos son observados y se utilizan generalmente, en la investigación causal estadística. Para efectos de este estudio, se contempla el sistema de educación superior en su conjunto heterogéneo, es decir, asumimos que el sistema aglutina las Universidades, Centros de Formación Técnica, Institutos Profesionales y Centros de Formación Militar.

\section{MARCO TEÓRICO}

\section{Inversión en Capital Humano y Retorno}

En la extensa literatura sobre retorno privado de la educación se ha demostrado de forma consistente que la educación es una buena inversión. La escolarización es un insumo importante en la construcción de capital humano y un año adicional de escolaridad tiende a aumentar el salario de un trabajador, por a lo menos, entre el $5 \%$ y el $15 \%$ (Canton 2007; Psacharopoulos y Patrinos 2004).

Por consiguiente, el cálculo del retorno en la educación se generalizó, encontrando importantes aportes de carácter empírico, como también la valorización de los beneficios no monetarios del capital humano y especialmente, la contribución de nuevos métodos econométricos. La abundante investigación sobre tasas de retorno de la inversión en educación, ha tenido una influencia crucial en dichas recomendaciones de política (Psacharopoulos y Patrinos 2004). La investigación en el área, ha retomado fuerza, en la medida que la educación ha pasado a ocupar un lugar estratégico de la política pública, en la mayoría de los países y, especialmente, debido a la potencial vinculación entre educación y desarrollo económico (Carnoy, 2011).

No obstante el trabajo de Hung (2008), cita los trabajos seminales de Becker (1964); Mincer (1974), y de Schultz (1961); desarrollan avances en la teoría del capital humano. El principio fundamental, detrás de esta teoría, fue la tasa privada y pública de los retornos a la educación y su impacto en la inversión y la financiación de la educación superior (Atuahene, 2007). Para Schultz, (1961) la educación puede ser considerada más que un bien de consumo, debido al mayor conocimiento de las personas que acceden a ella, las cuales alcanzan un mayor bienestar personal o estatus. Becker (1964), establece que el capital humano se sustenta en que las personas amplían sus capacidades como consumidores, al invertir en sí mismos, y que la escolaridad es la mayor inversión, los individuos acumulan capital humano con el fin de aumentar habilidades que son recompensadas en el mercado del trabajo. En tanto, Mincer (1974), extiende la teoría del capital humano, incluyendo los años posteriores al término de la escuela como una medida útil de la capacitación y experiencia en el puesto de trabajo.

Asimismo, en el trabajo de Psacharopoulos y Ying (1992), se argumentan tres razones por las cuales es importante examinar las relaciones entre ganancias y educación (Psacharopoulos y Patrinos 2004) . En primer lugar, las diferencias de salarios entre los diferentes tipos de educación muestran el premio de invertir en educación, el cual es asociado con una relativa escasez en el mercado de graduados. En segundo lugar, si estas ganancias son asociadas con los costos de la inversión en los diferentes tipos de educación, entonces se puede hacer un análisis costo-beneficio de la inversión en educarse. Finalmente, más allá de las consideraciones de eficiencia, el premio asociado a los diferentes niveles de educación es una evaluación de la equidad en una sociedad, por ejemplo, cómo el proveer educación contribuye al alivio de la pobreza y a la reducción en la desigualdad en el ingreso.

Sin embargo, hasta nuestros días, la economía de la educación ha experimentado un desarrollo acelerado, no estando exenta de importantes controversias entre los economistas, dando lugar a teorías alternativas, especialmente corrientes nacidas en Norteamérica, como las denominadas de filtro y segmentación, impulsada por Arrow 1973 (Kivinen, Hedman, y Kaipainen, 2007). Por otro lado, examinar la relación entre nivel de educación e ingresos ha sido objeto de estudio permanente de la teoría de capital humano, es así como en Chile, encontramos estudios que se han realizado en las áreas de economía y educación, que tienden a explicar la relación de salario y educación (Contreras, Melo, y Ojeda, 2005; Meller, 2010; Sapelli, 2011; Urzúa, 2012), cuyos los estudios empíricos concluyen que, el acceder y titularse de la educación superior, tiene retornos económicos positivos.

\section{Movilidad Social}

La Educación Superior es estratégica en la formación de capital humano avanzado y éste, a su vez, vital para una economía intensiva en conocimiento; pero también porque aquella es el instrumento más adecuado para la movilidad social, ya que existe una relación directa entre años y nivel de formación educacional e ingresos económicos para las personas (Keep y Mayhew, 2004). Por consiguiente, el grado de calificación de la fuerza 
de trabajo, la demanda y oferta de individuos, son elementos determinantes para explicar la dispersión salarial de la población. Así, la sociedad le otorga mayor importancia al acervo educacional, ya que éste supone posibilidades efectivas de ascenso en la escala laboral y de ingresos.

Ahora bien, la definición única de movilidad social es un debate abierto, especialmente, entre sociólogos y economistas, y tampoco es monolítica entre éstos últimos. Es así como, una corriente considera que la oscilación en torno a alguna definición de línea de pobreza o un cohorte de ingreso, no constituye movilidad social, siendo ésta simplemente la respuesta de los agentes ante shocks económicos, lo que lleva a definir la movilidad social como un concepto más de largo plazo (Contreras, Cooper, Herman, y Neilson, 2008).

La movilidad social, puede definirse como el cambio temporal de las posiciones de las personas en la estructura social jerárquica y explora los determinantes de este cambio (Torche y Wormald, 2004), es decir, genéricamente como cualquier cambio en el tiempo de los atributos sociales y económicos de un sujeto, sea ésta una persona, un hogar o un grupo social. Por lo tanto, para estudiar la movilidad social es necesario, en consecuencia, registrar la historia económica del sujeto correspondiente, o deducirla a partir de técnicas estadísticas especiales.

Por tanto, cuando hablamos de movilidad social o ascenso social, lo relacionamos directamente con la variación de la familia o individuo, en una categorización o estratificación socioeconómica, ésta puede ser entendida como movimientos horizontales o verticales y, a su vez, puede ser intergeneracional o intrageneracional. El estudio empírico de la movilidad social es más complicado que el de la estructura de ingresos, porque demanda información longitudinal que es más compleja de obtener, pues requiere encuestar a una misma muestra de hogares o personas a lo largo del tiempo (Behrman, 2011). Desde una perspectiva normativa, en la movilidad intergeneracional de ingresos, existe un creciente consenso en la literatura académica, que consiste en privilegiar la igualdad de oportunidades por sobre la igualdad de resultados (típicamente de ingresos), como el objetivo social relevante para orientar las políticas públicas en materia de equidad social (Nuñez y Miranda, 2008).

Finalmente, la movilidad de ingresos está relacionada con las políticas del gobierno, para reducir la pobreza y las brechas de desigualdad. La importancia de los eventos demográficos y económicos que afectan la movilidad debe ser examinado cuidadosamente (Castro, 2011). Chile es una de las economías con mayor desigualdad en el mundo(Contreras y Gallegos, 2007). A pesar de grandes avances en la reducción de la pobreza, y un aumento significativo en el gasto social, la desigualdad de ingresos sigue siendo un problema, ya que al realizar comparaciones con otras naciones, Chile se encuentra entre los países con mayor coeficiente de Gini (Castro, 2011). En concordancia con lo anterior, la segregación socioeconómica del sistema escolar puede ser un factor limitante para el grado de movilidad social (Nuñez y Miranda, 2008).

\section{Educación Superior en Chile}

La expansión de la educación superior, permite aumentar las posibilidades de los grupos de excluidos, pero dependiendo de otras condiciones de contexto como el desarrollo demográfico, económico y los factores del mercado de trabajo, pueden afectar los retornos individuales de los individuos(Tieben et al., 2013).

En Chile, según datos de la CASEN (2011), hay 1.032.299 personas en la educación superior, de las cuales 750.145 tienen entre 18 y 24 años de edad, mostrando un aumento de la cobertura bruta de un $39.7 \%$ a un $45.8 \%$, desde el año 2009, lo cual se ha traducido en la incorporación a la enseñanza superior en todos los segmentos, en especial, los sectores más pobres. (MIDEPLAN, 2011). La población de entre 18 y 24 años pertenecientes al primer quintil -ó el $20 \%$ más pobre del país- es el que más ha avanzado en cobertura. En 1990 , el 15,7\% de los estudiantes de educación superior provenía de los dos primeros quintiles, hoy alcanza el 31,3\%, es decir, el $40 \%$ de los más pobres duplicó su participación en la educación superior, en dos décadas. Sin embargo, se sugiere cautela respecto de una positiva interpretación del aumento en las tasas de matrícula del sistema. Si bien el sistema de educación superior, ha generado una creciente primera generación de participantes, el análisis de las tasas de deserción y graduación sugiere que, dichas posibilidades, pueden no haberse manifestado en un mejor futuro.(Urzúa, 2012)

Por ello, la creciente matrícula en el sistema de educación superior, ha sido interpretada como un signo positivo y es un reflejo de la nueva movilidad social en Chile. El paso por una institución de educación superior, ha sido interpretado como el antídoto a la transmisión intergeneracional de la pobreza (Urzúa, 2012). El mismo autor, enfatiza que la encuesta CASEN (2009), revela que un 64\% de los adultos jóvenes con estudios superiores (221.356 de 344.659), provenía de familias en que los padres habían alcanzado como máximo un título de educación media, todo un éxito desde el punto de vista de la movilidad social. 
A pesar que, el sistema de educación superior, ha ampliado las oportunidades para egresados provenientes de estratos más vulnerables de la población, la sociedad chilena ha mostrado su fuerte desigualdad (Sapelli, 2009), principalmente expresada en la alta predictibilidad que muestran sus resultados en la transmisión intergeneracional, siendo el factor "cuna de origen del estudiante", determinante sobre los resultados en el ciclo escolar y, con mayor fuerza, en el ingreso y permanencia en la educación superior (Donoso, 2009).

En Chile, distintos estudios concluyen que, el acceder y titularse de la educación superior tiene retornos económicos positivos (Contreras y Gallegos, 2007; Meller, 2010; Sapelli, 2009, 2011; Urzúa, 2012). Del informe de la CASEN (2011), se puede observar que el mayor crecimiento de los ingresos se produce en los niveles de escolaridad igual o superior a 12 años, estableciendo una relación positiva entre el nivel de ingresos del trabajo declarado y los años de escolaridad de cada persona, inclusive desde la educación superior incompleta (MIDEPLAN, 2011).

Usando datos de la CASEN, Sapelli (2009) estima que un individuo con estudios terciarios alcanza, en promedio, un ingreso de $25 \%$ más alto de lo que obtendría sin éstos. Asimismo, encuentra que el último año de educación universitaria tendría un retorno cercano al 40\%, mientras que los años anteriores tendrían un retorno del $20 \%$. Lo anterior, es coherente con la idea de que obtener un título también es un indicador de señalización en el mercado. En tanto, Urzúa (2012), utilizando información de los aranceles de las carreras según institución, estima las tasas de retorno para cada una de éstas. Sus principales conclusiones, muestran que existe una alta heterogeneidad en los aranceles y retornos de las carreras, inclusive habría varias instituciones con retornos negativos, por lo cual, el autor llama a mirar con mayor cautela el retorno de la educación superior. Este trabajo es coincidente con los estudios anteriores de Benavente et al., (2005), donde se muestra que un conjunto de carreras universitarias presentan diferencias significativas de remuneraciones, dependiendo de la institución en la cual se obtuvo el título correspondiente (Meller y Rappoport, 2008).

Por consiguiente, los distintos estudios apoyan la hipótesis de que la educación superior tiene una elevada rentabilidad en nuestro país, y esto explica en parte, el alto interés por aumentar su cobertura. Sin embargo, se debe tener en cuenta la carrera, la institución y, lo más importante, completar los estudios. El sistema de educación superior contribuye a la movilidad social, toda vez que éste sea de calidad. Por tanto, resulta crucial resguardar la calidad, particularmente, dentro de los procesos formadores de capital humano (Pedraja et al. 2012).

\section{METODOLOGÍA}

Para determinar lo anterior, se utilizará el estimador de diferencias en diferencias, cuyo objetivo es evaluar el impacto de la educación superior en el ingreso de un grupo de individuos, para lo cual, se asume la existencia de dos grupos, en una condición base y estadísticamente comparables entre sí. Primero, un grupo que técnicamente es el que recibe educación superior (experimento) y el otro grupo, que no recibe educación superior (grupo control o no tratado), se comparan en dos periodos de tiempo y se calcula porcentualmente, la diferencia de ingresos entre los individuos que recibieron educación superior respecto a aquellos que no recibieron y que estadísticamente, son comparables. Por consiguiente, se plantea trabajar con dos grupos, el "no tratado" que no es beneficiario, y el "grupo tratado", que es beneficiario (Blundell, Dearden, y Sianesi, 2005). Para tales efectos, se utilizará el software estadístico Stata, obteniendo la variable $\beta_{3}$. Un valor positivo del estimador de diferencias en diferencias, permite evaluar favorablemente los efectos sobre la población beneficiaria.

Grupo "Tratado" $(E)$ = Conjunto de individuos tratados o beneficiarios de "Educación Superior", lo que comprende Universidades, Institutos Profesionales, Centros de Formación Técnica incluyendo Centros de Formación Militar.

Grupo de "no Tratado" (C) = Conjunto de Individuos no tratados con "Educación Superior", lo que comprende, sin educación formal, básica incompleta, básica completa, media $\mathrm{C} / \mathrm{H}$ incompleta, media $\mathrm{C} / \mathrm{H}$ completa, media T/P incompleta, media T/P completa, C.F.T/I.P incompleta, Universidad incompleta, sin dato.

Variables a considerar:

$Y_{T+1}^{E}=$ Ingreso autónomo promedio del individuo i perteneciente al grupo tratado en período $\mathrm{t}+1$.

$Y_{T}^{E}=$ Ingreso autónomo promedio del individuo i perteneciente al grupo tratado en período t.

$Y_{T+1}^{C}=$ Ingreso autónomo promedio del individuo i, que no es tratado en período $\mathrm{t}+1$.

$Y_{T}^{C}=$ Ingreso autónomo promedio del individuo i, que no es tratado en el periodo t.

Así, tendremos entonces el siguiente estimador: 
1) $\alpha=\left(Y_{T+1}-Y_{T}\right)^{E}-\left(Y_{T+1}-Y_{T}\right)^{C}$, con especificación econométrica :2) $Y_{i}=\beta_{0}+\beta_{1} X_{i}+\beta_{2} Z_{i}+\beta_{3} X_{i} Z_{i}+\varepsilon$ donde:

$X_{i} \Rightarrow\left\{\begin{array}{l}1 \text { Si el individuo es del periodo } T+1 \\ 0 \text { Si el individuo es del periodo } T\end{array} \wedge Z_{i} \Rightarrow\left\{\begin{array}{l}1 \text { Si el individuo es del grupo Experimental }(E) \\ 0 \text { Si el individuo es del grupo Control }(C)\end{array}\right.\right.$

Por tanto:

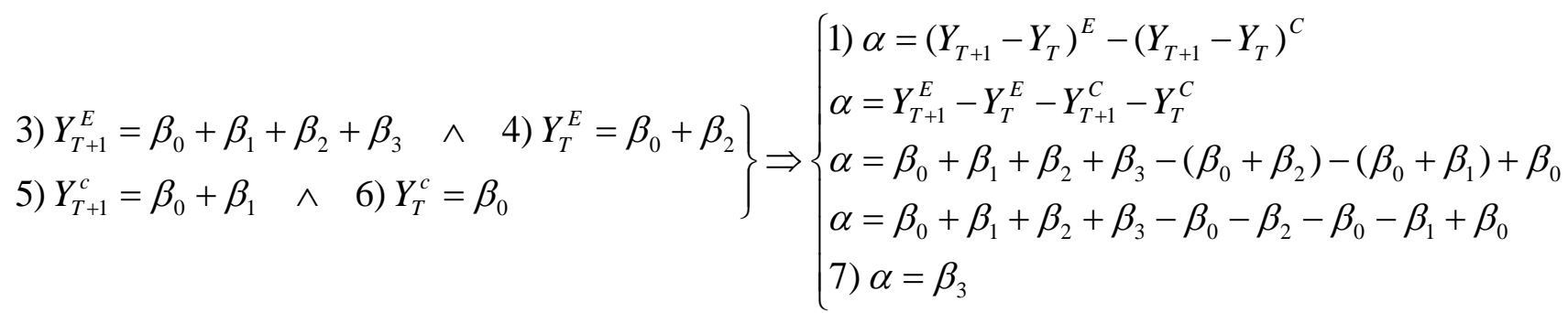

Así la ecuación 7) $\alpha=\beta_{3}$ nos indicará el impacto en el individuo, al ser beneficiario del tratamiento. En este modelo, consideraremos la muestra total, es decir, los grupos tratados y no tratados y luego aplicamos log al Ingreso $(Y)$ dado que, el resultado de los estimadores de la regresión, corresponderá a semielasticidades (variaciones porcentuales).

8) $\log \left(Y_{i}\right)=\beta_{0}+\beta_{1} X_{i}+\beta_{2} Z_{i}+\beta_{3} W_{i i}+\varepsilon$

donde $\Rightarrow\left\{\begin{array}{l}W_{i}=X_{i} \bullet Z_{i} \\ \beta_{3}=\operatorname{Impacto}(\%) \text { de la Educación Superior en el ingreso del Individuo. }\end{array}\right.$

\section{RESULTADOS Y DISCUSIÓN}

La población considerada comparativamente, se divide en dos grandes grupos, los cuales se contrastan en un espacio determinado de tiempo, que a su vez, se subdividen en otros dos grupos (tratado y no tratado). Por tanto, al momento de compararlos en dichos intervalos y al aplicar el tratamiento, se encuentran dos realidades diferentes, en las cuales el factor común es la movilidad social intrageneracional, esto se debe básicamente porque existen desplazamientos en la estructura de ingresos, dentro de la población, si tomamos nuestra estructura de ingresos y describimos su trayectoria en un intervalo temporal, es posible determinar en qué proporción se movieron los datos y hacia dónde. La tabla N.1, muestra las salidas para el intervalo 2003-2006.

Tabla 1: Tratamiento CASEN2003 - CASEN2009

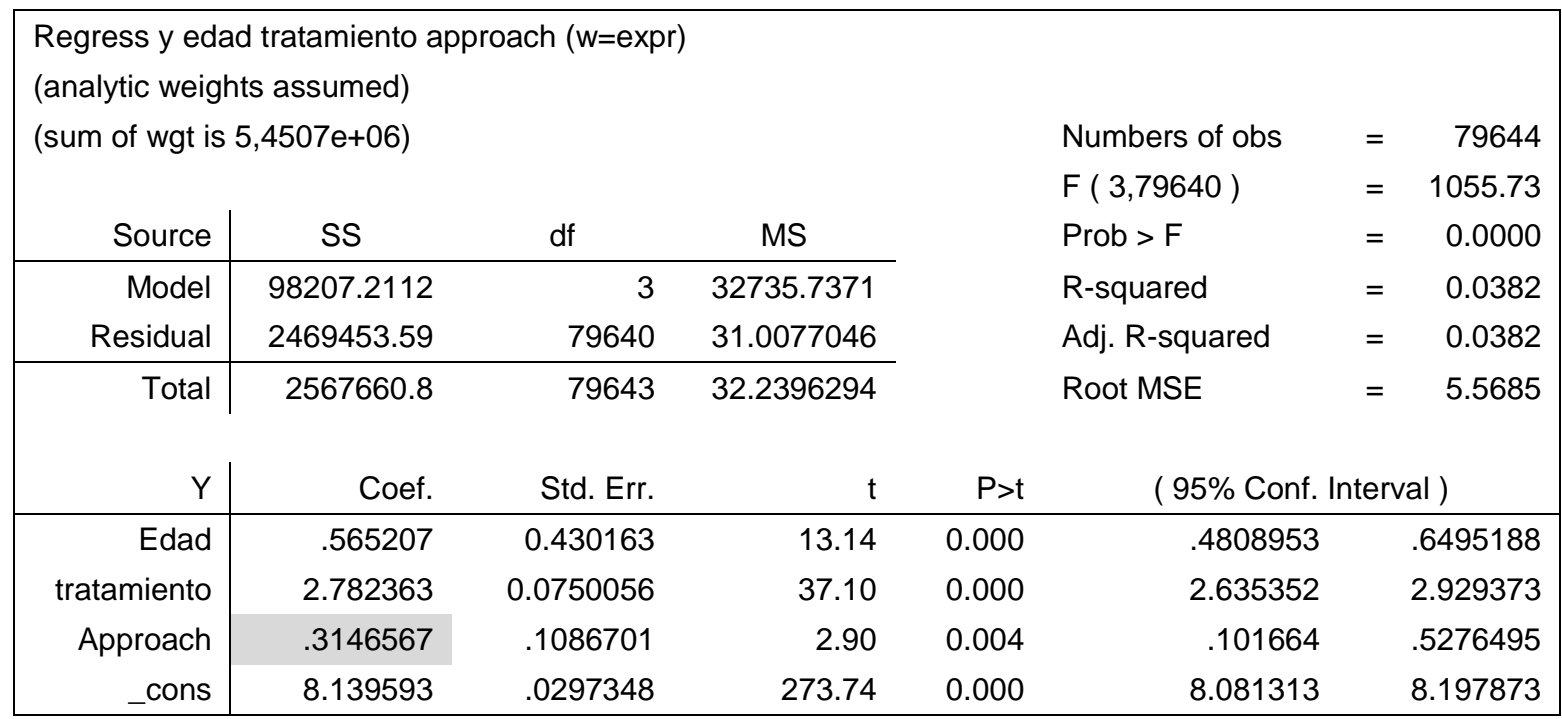


A partir del análisis estadístico realizado con Stata, se obtiene el impacto en Educación Superior de 31,47\% (destacada en tabla 1) y cuyos parámetros se expresan en la ecuación siguiente:

$$
\log \left(Y_{i}\right)=\beta_{0}+\beta_{1} X_{i}+\beta_{2} Z_{i}+\beta_{3} W_{i i}+\varepsilon \Rightarrow \log \left(Y_{i}\right)=8,1396+0,5652 X_{i}+2,7824 Z_{i}+0,3147 W_{i}+\varepsilon
$$

Bajo las condiciones del cálculo, podríamos establecer una aproximación a movilidad social intrageneracional: $\alpha=\beta_{3}=31.47 \%$

El resultado del tratamiento, para este caso e intervalo de proyección, indica implícitamente que posee un efecto positivo en los individuos alcanzar estudios superiores y, esto se ve reflejado directamente, en sus ingresos futuros. De lo anterior, se obtiene una predicción de nuestra función, cuya interpretación permite apreciar una relación netamente positiva, en cuanto se incrementan los años de estudios y el impacto que esto tiene en los ingresos.

Intervalo 2006-2011

Para los efectos del estudio, la política es la obtención de título en educación superior, proyectado en el tiempo, para lo cual también hemos simulado para el intervalo 2006-2011, cuyo tratamiento se muestra en tabla 2.

Tabla 2: Tratamiento CASEN2006 - CASEN2011

\begin{tabular}{|c|c|c|c|c|c|c|}
\hline \multirow{2}{*}{\multicolumn{5}{|c|}{$\begin{array}{l}\text { Regress y edad tratamiento approach ( } \mathrm{w}=\mathrm{expr} \text { ) } \\
\text { (analytic weights assumed) } \\
\text { (sum of wgt is } 5,3482 \mathrm{e}+06)\end{array}$}} & \multirow{3}{*}{$\begin{array}{l}\text { Numbers of obs } \\
F(3,79640) \\
\text { Prob > F }\end{array}$} & \multirow{3}{*}{$\begin{array}{rr}= & 72430 \\
= & 1220.28 \\
= & 0.0000\end{array}$} \\
\hline & & & & & & \\
\hline Source & SS & df & MS & & & \\
\hline Model & 106329.214 & 3 & 35443.0714 & & R-squared & $=\quad 0.0481$ \\
\hline Residual & 2103615.74 & 72426 & 29.0450355 & & Adj. R-squared & 0.0481 \\
\hline Total & 2209944.95 & 72429 & 30.5118799 & & Root MSE & 5.3893 \\
\hline $\mathrm{Y}$ & Coef. & Std. Err. & $\mathrm{t}$ & $P>t$ & \multicolumn{2}{|c|}{ ( $95 \%$ Conf. Interval ) } \\
\hline Edad & .655801 & .0452094 & 14.51 & 0.000 & .5671908 & .7444112 \\
\hline tratamiento & 2.941023 & .0672859 & 43.71 & 0.000 & 2.809143 & 3.072903 \\
\hline Approach & -.154676 & .0985572 & -1.57 & 0.117 & -.3478478 & .0384958 \\
\hline cons & 8.455505 & .0306442 & 275.93 & 0.000 & 8.395442 & 8.515567 \\
\hline
\end{tabular}

A partir del análisis estadístico realizado con Stata, se obtiene el impacto en Educación Superior de -15,47\% (destacada en tabla 2) y cuyos parámetros se expresan en la ecuación siguiente:

$$
\log \left(Y_{i}\right)=8,4555+0,6558 X_{i}+2,9410 Z_{i}+0,1547 W_{i}+\varepsilon
$$

Bajo las condiciones del cálculo, podríamos establecer una aproximación a movilidad social intrageneracional: $\alpha=\beta_{3}=-15.47 \%$

Lo cual para este caso, indica implícitamente que la política aplicada, tratamiento o beneficio en la proyección de los datos establecidos, no posee un efecto positivo en el ingreso, siendo nulo. Por otra parte, la movilidad social sigue estando presente, su valor absoluto representa la proporción del movimiento dentro de la estructura de ingresos y, su signo explica que los salarios disminuyeron, en términos reales. El método de estimación de diferencias en diferencias, contempla una mayor cobertura, lo cual puede ser cuestionable, ya que se refiere al conjunto heterogéneo que compone el sistema de educación superior.

Para el primer caso, el tramo 2003-2006, la evaluación sería favorable, dado su resultado positivo, pero a la vez, si bien existe movilidad social en promedio, ésta puede ser cuestionable debido al nivel de desigualdad que existe en Chile y la marcada segregación al interior de la población, coherente con los hallazgos de Contreras et al., (2005). En el segundo caso, para los años 2006-2011, existe un impacto nulo en los ingresos de los individuos, lo cual es convergente con el trabajo de Urzúa, (2012), donde el incremento de cobertura en educación superior, por sí solo, no cambia la redistribución del ingreso de forma significativa, dada la 
heterogeneidad de instituciones y carreras, las cuales influyen en las remuneraciones futuras de sus egresados, incorporando indicadores de selectividad y monitoreo.

Finalmente, se reafirma en el modelo chileno que, la desigualdad de ingresos y educación, es concebida desde la pre-infancia y asociada a sus factores de origen familiar ( Behrman, 2011; Carneiro y Heckman 2003), por ello que los retornos más altos se relacionan con el financiamiento en los primeros años de vida. La mayor movilidad intergeneracional de la educación, no se estaría traduciendo necesariamente en una mayor movilidad intergeneracional del ingreso, en las últimas décadas, lo que sugiere la existencia de factores que inhiben o limitan la transformación de mayor escolaridad, en mejores perspectivas de generación de ingresos (Nuñez y Miranda, 2008).

\section{CONCLUSIONES}

Al calcular el estimador de "diferencias en diferencias", en el contexto de estudios observacionales, podemos afirmar que, al considerar el intervalo 2003-2009, el estimador muestra un resultado positivo. Es decir, en promedio, el incremento en el nivel de ingresos autónomos de los individuos beneficiados por la política o premio de la obtención al título de educación superior, expresado en términos porcentuales, es el equivalente a un $31,47 \%$, superior al incremento que registran los individuos del "grupo no tratado", entre los momentos previos y posteriores de aplicado el beneficio.

En tanto, nuestros resultados muestran que en el intervalo 2006-2011, la tasa interna de retorno de la educación superior proyectada y comparada en dichos años, no mejora la situación del individuo, mostrando una valor de $-15,47 \%$, existiendo una pérdida del poder adquisitivo. Una tasa interna de retorno negativa, por consiguiente, significa que no es rentable estudiar algunas de las carreras de la parrilla ofrecida por las instituciones educativas.

\section{REFERENCIAS}

Abadie, A., Semiparametric Difference-in-differences Estimators. Review of Economic Studies, 72(1), 1-19 (2005).

Altbach, P., Reisberg, L., y Rumbley, L., Trends in global higher education: Tracking an academic revolution. UNESCO Pub. Francia (2009).

Atuahene, F., The challenge of financing higher education and the role of student loans scheme: an analysis of the student loan trust fund (SLTF) in Ghana. Higher Education, 56(4), 407-421.

Behrman, J. R., How much might human capital policies affect earnings inequalities and poverty? Estudios de Economía, 38(1), 9-41 (2011).

Blundell, R., Dearden, L., y Sianesi, B., Evaluating the effect of education on earnings: models, methods and results from the National Child Development Survey. Journal of the Royal Statistical Society: Series A (Statistics in Society), 168(3), 473-512 (2005).

Canton, E., Social Returns to Education: Macro-Evidence. De Economist, 155(4), 449-468 (2007).

Carneiro, P., y Heckman, J., Human capital policy. In B. W. Friedman (Ed.), Inequality in America: What role for human capital policies? Cambridge,MA: MIT Press., 77-239 (2003).

Carneiro, P., Heckman, J., y Vytlacil, E., Estimating Marginal Returns to Education. National Bureau of Economic Research, 1-32 (2010).

Carnoy, M., As Higher Education Expands, Is It Contributing To Greater Inequality? National Institute Economic Review, 215(1), R34-R47 (2011).

Castro, R., Getting ahead, falling behind and standing still . Income mobility in Chile. Estudios de Economía, 38(1), 243-258 (2011).

Contreras, D., Cooper, R., Herman, J., y Neilson, C., The Dynamics of Poverty in Chile. Journal of Latin American Studies, 40, 251-273 (2008).

Contreras, D., y Gallegos, S., Descomponiendo la desigualdad salarial en América Latina: ¿Una década de cambios? Serie de estudios estadísticos y propectivos - CEPAL, 59, 1-46 (2007).

Contreras, D., Melo, E., y Ojeda, S., ¿ Estimando el retorno a la educación o a los no observables? Evidencia de datos de panel. Estudios de Economía, 32(2), 187-199 (2005). 
Donoso, S., Economía política del financiamiento de los estudios universitarios en Chile (1980-2010): debate de sus fundamentos. Innovar, especial e, 141-156 (2009).

Heckman, J., Ichimura, H., Smith, J., y Todd, P., Characterizing Selection Bias Using Experimental Data. Econometrica, 66(5), 1017-1098 (1998).

Hung, F., Returns to education and economic transition: an international comparison. Compare: A Journal of Comparative and International Education, 38(2), 155-171 (2008).

Keep, E., y Mayhew, K., The economic and distributional implications of current policies on Higher Education. Oxford Review of Economic Policy, 20(2) (2004).

Kivinen, O., Hedman, J., y Kaipainen, P., From Elite University to Mass Higher Education: Educational Expansion, Equality of Opportunity and Returns to University Education. Acta Sociologica, 50(3), 231-247 (2007).

Meller, P., Carreras Universitarias: Rentabilidad, Selectividad y Discriminación. (Uqbar edit.) (2010).

Meller, P., y Rappoport, D., ¿Son siempre las universidades la mejor opción para un título profesional? Evidencia chilena. Trimestre económico, 75(4), 897-929 (2008).

MIDEPLAN, Encuesta Casen. Retrieved from www.mideplan.cl/casen (2008)

Mincer, J., Schooling, Experience and Earning. New York Columbia University Press (1974).

Moretti, E., Estimating the social return to higher education: evidence from longitudinal and repeated crosssectional data. Journal of Econometrics, 121(1-2), 175-212 (2004).

Münich, D., Svejnar, J., y Terrell, K., Returns to Human Capital under the Communist Wage Grid and during the Transition to a Market Economy. Review of Economics and Statistics, 87(1), 100-123 (2005).

Nuñez, J., y Miranda, L., Trends in Intergenerational Income and Educational Mobility: The case of Chile. Mimeo, Departamento de Economía, Universidad de Chile (2008).

Pedraja, L., Araneda, C., Rodríguez, E., y Rodríguez, J., Calidad en la Formación Inicial Docente : Evidencia Empírica en las Universidades Chilenas. Formación universitaria, 5(4), 15-26 (2012).

Psacharopoulos, G., y Patrinos, H. A., Returns to investment in education: a further update. Education Economics, 12(2), 111-134 (2004).

Sapelli, C, Los retornos a la educación en Chile: estimaciones por corte transversal y por cohortes. Documento de Trabajo PUC, 349, 1-77 (2009).

Sapelli, C., A cohort analysis of the income distribution in Chile. Estudios de Economía, 38(1), 223-242 (2011).

Tieben, N., Hofäcker, D., y Biedinger, N., Social mobility and inequality in the life course: Exploring the relevance of context. Research in Social Stratification and Mobility, 32, 1-6. doi:10.1016/j.rssm.2013.03.002 (2013)

Torche, F., y Wormald, G., Estratificación y movilidad social en Chile: entre la adscripción y el logro. Serie de Políticas Sociales, No 98 CEPAL, Santiago (2004).

Urzúa, S., La rentabilidad de la Educación Superior en Chile ¿Educación Superior para todos? Documento de Trabajo, Centro de Estudios Públicos, Nro. 386, 1-43 (2012). 
